\title{
Improved Canny Algorithm for Edge Detection of Core Image
}

\author{
Tian-Shi Liu*, Rui-Xiang Liu*, Ping-Zeng and Shao-Wei Pan
}

School of Computer Science, Xi'an Shiyou University, Xi'an 710065, China

\begin{abstract}
Aiming at the disadvantages of edge detail loss and false edges in conventional Canny algorithm, this paper proposes an optimized Canny edge detection algorithm for edge detection of core image by combining self-adaption smooth filtering and self-adaptive threshold value. The algorithm, which retains the advantages of high signal to noise ratio, accurate positioning, single edge single response and etc in conventional Canny algorithm, has stronger de-noising capability and better edge-detection effect. The experiment results indicate that the improved algorithm can perform selfadaptive edge-detection on the core image and create a clear coherent detected edge of image which can display the edge feature better.
\end{abstract}

Keywords: Canny algorithm, Core image, edge detection, image de-noising.

\section{INTRODUCTION}

The core data is one of the critical and intuitive information in studies of oil exploration and development. Identification and analysis of core image are the main work of indepth analysis of petroleum geology [1]. However, due to the complexity of interference sources and the relatively large noise of the core image, analysis and study for the core image will not be carried out effectively without going through the effective noise elimination processing. Denoising of core image becomes the key of core image processing. The edge of image is the important information of image feature and the foundation of characteristic shape analysis. In order to achieve accurate positioning of the target image, we need to detect the edge of the core image on the basis of de-noising. The result of edge detection will affect the understanding and recognition performance of image directly. In the method of classic edge detection, the commonly used first-order differential operators are Robets operator, Prewwits operator, and Sobel operator. Commonly used two order differential operators are Laplace operator and Log operator. These operators have some features: simple structure, easy to implement, good real-time. But they are sensitive to the impact of noise. In addition, they have a poor anti-interference performance, lower edge precision and rough edge $[2,3]$.

In order to improve accuracy of human behavior recognition, the Canny algorithm was combined with the model of human behavior recognition of neural network in the reference [4] which used Canny algorithm to pretreat the human behavior images, trained neural network models that can recognize human behavior model and carry out the simulat- ion experiment. The reference [5] proposed a method for image edge detection which was based on the technology of Canny algorithm and image morphology fusion cross-use. It applied Canny algorithm and the image morphology to detect the image edge, fused the images of the two results produced by those two detecting methods using the wavelet fusion technology, and obtained the final edge-image. These algorithms mentioned above make up for the deficiency of Canny algorithm, but increase the complexity of the algorithm. In order to extract the edge of core image better, based on reference [6-8], this paper proposes an optimized Canny edge detection algorithm by combining self-adaption smooth filtering and self-adaptive threshold value. Comparing with conventional differentiating operator, this algorithm can inhibit noise effectively, reduce disturbing influence of edge, as well as display edge feature of the core image well.

\section{CANNY ALGORITHM OF EDGE DETECTION}

\section{A. Criterion}

Canny algorithm is an idea based on the optimization of ideology which proposes three criteria for evaluation of the performance of edge detection [9, 10]:

\section{1) High SNR (Signal-to-Noise Ratio) criterion}

It focuses on good test results. It makes the minimum probability in which the non-edge points sentence the edge points, or the edge points sentence the non-edge points. The greater the SNR is, the higher the quality of the extracted edge is.

2) High positioning accuracy criterion

It emphasizes the minimum distance between detected edge point and the actual edge point so that the position accuracy of the edges is the highest. 


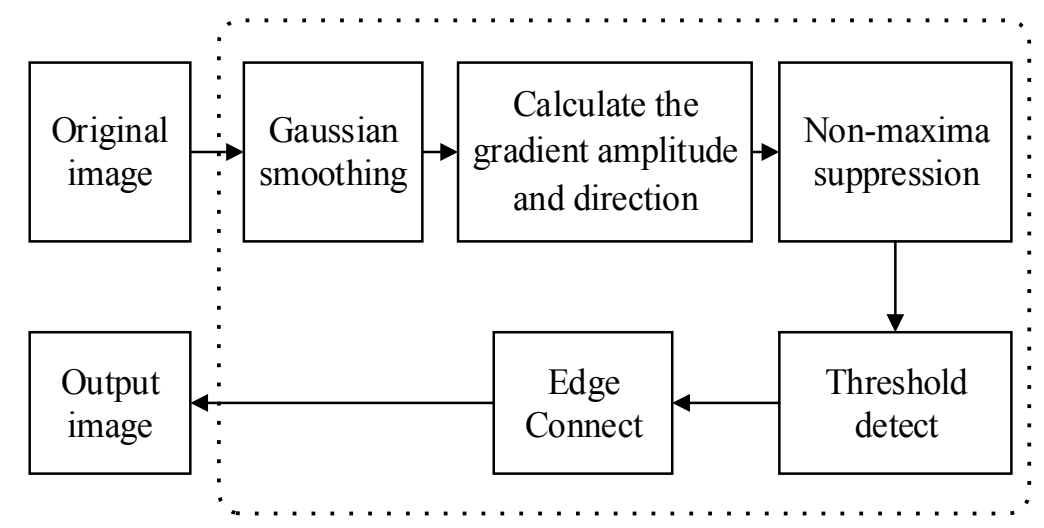

Fig. (1). Canny algorithm implementation process.

3) Single-edge response criterion

It responses only one time for a single edge point, and inhibits the false edges maximally.

\section{B. The Implementation of Traditional Canny Algorithm}

The implementation of traditional Canny algorithm mainly includes four parts: smooth image, the calculation of the amplitude and direction of the gradient, inhibition of nonmaxima for the gradient amplitude, detection with dualthreshold method and edge connection [10], shown as in Fig. (1).

\section{1) Smooth images}

Select two-dimensional Gaussian function $\mathrm{G}(\mathrm{x}, \mathrm{y})$ to construct filters, and obtain smooth images $\mathrm{I}(\mathrm{x}, \mathrm{y})$ by the convolution of the original image $f(x, y)$ according to the row and column respectively.

$G(x, y)=\frac{1}{2 \pi \sigma^{2}} \exp \left[-\frac{x^{2}+y^{2}}{2 \sigma^{2}}\right]$

$I(x, y)=G(x, y) * f(x, y)$

Where, ' $\sigma$ ' is the standard deviation of Gaussian function and used to control the degree of smoothness. The exported expression is similar to the second derivative of Gaussian function, and contains the product of edge location accuracy and signal-to-noise ratio by using functional derivative method based on the above three principles. Consequently, the two-dimensional Gaussian function is used to smooth the image [11].

2) Calculate the gradient amplitude and direction [12].

Gradient calculation usually adopts the method of partial derivatives. Canny algorithm uses the finite difference of first order partial derivative of $2 \times 2$ neighborhood to calculate gradient amplitude $\mathrm{M}(\mathrm{x}, \mathrm{y})$ and gradient direction $\mathrm{H}(\mathrm{x}, \mathrm{y})$ of the smoothed image $\mathrm{I}(\mathrm{x}, \mathrm{y})$ :

$\mathrm{M}(\mathrm{X}, \mathrm{Y})=\sqrt{\mathrm{k}_{\mathrm{x}}^{2}(\mathrm{x}, \mathrm{y})+\mathrm{k}_{\mathrm{y}}^{2}(\mathrm{x}, \mathrm{y})}$

$$
\begin{aligned}
& H(x, y)=\arctan \left[k_{x}(x, y), k_{y}(x, y)\right] \\
& f_{x}=\left[\begin{array}{ll}
-0.5 & 0.5 \\
-0.5 & 0.5
\end{array}\right], f_{y}=\left[\begin{array}{cc}
0.5 & 0.5 \\
-0.5 & -0.5
\end{array}\right]
\end{aligned}
$$

Where $\mathrm{k}_{\mathrm{x}}$ and $\mathrm{k}_{\mathrm{y}}$ are the results which are the effects of filter $f_{x}$ and filter $f_{y}$ on the original image $I(x, y)$ along the row and column respectively.

3) Non-maximum suppression for the gradient amplitude

The gradient amplitude is interpolated along the gradient direction for all elements of $\mathrm{M}(\mathrm{i}, \mathrm{j})$ in the neighborhood of 8 directions with $3 \times 3$ window. For each point, the central element $\mathrm{M}(\mathrm{i}, \mathrm{j})$ in the neighborhood is compared with the two interpolation results of gradient amplitude along the gradient direction. The corresponding edge flag of $\mathrm{M}(\mathrm{i}, \mathrm{j})$ is 0 if the value of $M(i, j)$ is less than the two interpolation results. tion

4) Double threshold method detection and edge connec-

The gradient amplitude of non-maximum suppression is processed by using the high threshold $\mathrm{H}$ and the low threshold L. The gray level value of pixel whose gradient is less than the threshold is 0 . The two threshold edge images $\mathrm{H}(\mathrm{i}, \mathrm{j})$ and $\mathrm{L}(\mathrm{i}, \mathrm{j})$ are obtained by segmentation. When the connecting edge profile of the image $\mathrm{H}(\mathrm{i}, \mathrm{j})$ is connected to the endpoint, the weak edge points of the image $L(i, j)$ can be sought and the gap of edge of the image $\mathrm{H}(\mathrm{i}, \mathrm{j})$ can be filled. Thus, the determination of high and low thresholds refers to the substantive issues of edge detection. Therefore, the selection of the proper high and low thresholds is the key to the Canny algorithm.

\section{IMPROVED IMAGE EDGE DETECTION AL- GORITHM}

\section{A. Adaptive Smooth Filtering Algorithm}

The traditional Canny algorithm generally uses Gaussian filter to smooth image, which adjusts the smoothness of the image by changing the standard deviation value ' $\sigma$, of 
Gaussian. However, because the Gaussian filter smoothes all gray level value of pixels in the image and doesn't reserve the mutative gray level value of pixel, the edge points of the image are smoothed out [13]. In order to retain the image edges better and avoid the smoothness of the mutative pixel, the weight of the Gaussian filter is set to 0 at the mutative position of pixel gray level value. The proper selection of the filter window can avoid averaging two different regions which are separated by mutation. Moreover, the mutative pixel is attributed to the neighbor region in the process of smoothness. Consequently, the image edges will be enhanced by smooth filtering.

However, the mutation position of the image pixel is practically unknown and the steepness of the mutation position also is not clear. Consequently, the weight of any point cannot be simply set to 0 or 1 . In order to solve this problem, the weight coefficient of the filter is adjusted adaptively by calculating discontinuously the gray level value of the original image pixel [14].

The filter uses iterative convolution of the local weighted template and the original image signals to obtain the smooth image. Its iterations are based on the optimal iteration stopping criteria. This process has the nature of anisotropic diffusion. The weight coefficient of each pixel is changing in the process of iteration, which reflects the degree of continuity of the gray level value.

\section{B. The Adaptive Threshold Algorithm}

In the Canny algorithm, the selection of threshold is very critical. If the threshold is selected properly, it not only can reduce noise, but also has a good effect to suppress the pseudo-edges. The edge will be interrupted and discontinuous if the threshold is set too high. Otherwise, excessive pseudoedge may be retained and noise also may be used as the edge if the threshold is set too low in the process of extracting edge. The traditional Canny algorithm needs to set the high and low thresholds artificially by experience, which may need to be repeatedly tested to find a appropriate threshold, and the ratio between high threshold and low threshold is a constant value. However, the actual images are affected easily by light, scenes and other uncertain factors. The proportion of optimum threshold is not a constant value for different images. Consequently, the traditional Canny algorithm has unavailability in selecting the threshold. Therefore, this paper uses an adaptive method to automatically calculate the dual-threshold and selects the most appropriate threshold objectively and correctly by combining the characteristics of the image [8].

\section{Improved algorithm description}

The concrete steps of the improved Canny algorithm is described as follows:

Step1: Read the detected core image

Step2: According to the filter window size, use the method of adaptive smooth filtering to process the image. The processes are described as follows:

1) Set iterations $k=0$
In this paper, the selection of the iterations $\mathrm{K}$ is based on iteration stopping criterion of the anisotropic diffusion filter. The optimal iteration stopping time is obtained when the correlation of the smoothed image and the noise is the minimum value $[15,16]$. The correlation coefficient is calculated by:

$\rho_{u_{0}-u_{t}, u_{t}}=\frac{\operatorname{cov}\left(u_{0}-u_{t}, u_{t}\right)}{\sqrt{\operatorname{var}\left(u_{0}-u_{t}\right)} \sqrt{\operatorname{var}\left(u_{t}\right)}}$

Where, $u_{0}$ represents the original image. $u_{t}$ represents the filtered image at time $t . u_{0}-u_{t}$ represents the image noise. The iterations $\mathrm{K}$ is optimal when the correlation coefficient is minimum.

2) Calculate the gradient $G_{m}^{(k)}(m, n)$ and $G_{n}^{(k)}(m, n)$

$$
\begin{aligned}
& \mathrm{G}_{\mathrm{m}}^{(\mathrm{k})}(\mathrm{m}, \mathrm{n})=\frac{1}{2}\left[\mathrm{f}^{(\mathrm{k})}(\mathrm{m}+1, \mathrm{n})-\mathrm{f}^{(\mathrm{k})}(\mathrm{m}-1, \mathrm{n})\right] \\
& \mathrm{G}_{\mathrm{n}}^{(\mathrm{k})}(\mathrm{m}, \mathrm{n})=\frac{1}{2}\left[\mathrm{f}^{(\mathrm{k})}(\mathrm{m}, \mathrm{n}+1)-\mathrm{f}^{(\mathrm{k})}(\mathrm{m}, \mathrm{n}-1)\right]
\end{aligned}
$$

3) Calculation the weights of filter

$\mathrm{w}^{(\mathrm{k})}(\mathrm{m}, \mathrm{n})=\operatorname{cxp}\left[-\frac{\left[\mathrm{G}_{\mathrm{m}}^{(\mathrm{k})}(\mathrm{m}, \mathrm{n})\right]^{2}+\left[\mathrm{G}_{\mathrm{n}}^{(\mathrm{k})}(\mathrm{m}, \mathrm{n})\right]^{2}}{2 \mathrm{~h}^{2}}\right]$

4) The weighted average of $f^{(k)}(m, n)$

$$
f^{(k+1)}(m, n)=\frac{\sum_{i=-1}^{1} \sum_{j=-1}^{1} f^{(k)}(m+i, n+j) w^{(k)}(m+i, n+j)}{\sum_{i=-1}^{1} \sum_{j=-1}^{1} w^{(k)}(m+i, n+j)}
$$

5) If $\mathrm{k}=\mathrm{K}$, the iteration end. Otherwise $\mathrm{k}=\mathrm{K}+1$, and go to step (2)

Step3: Calculate the amplitude and direction of gradient

The gradient magnitude is calculated by using the first order difference for image.

Step4: Inhibit the non maxima of gradient magnitude

The gradient amplitude is interpolated along the gradient direction of the gradient amplitude. The maximum value is selected as candidate edge points and pixel point of the non maximum is set to 0 .

Step5: Calculate the dual-threshold.

The adaptive algorithm is adopted to obtain the optimal threshold. The specific process of the adaptive threshold algorithm is described as follows: Assuming the number of pixels of the image is $M$. The range of the gray level value is $[0, K]$. The pixel number is $n_{i}$ when the gray level value is $\mathrm{i}$. The pixels of the image are divided into two groups $\mathrm{A}$ and $\mathrm{B}$ according to the gray level threshold value $\mathrm{t}$. A 

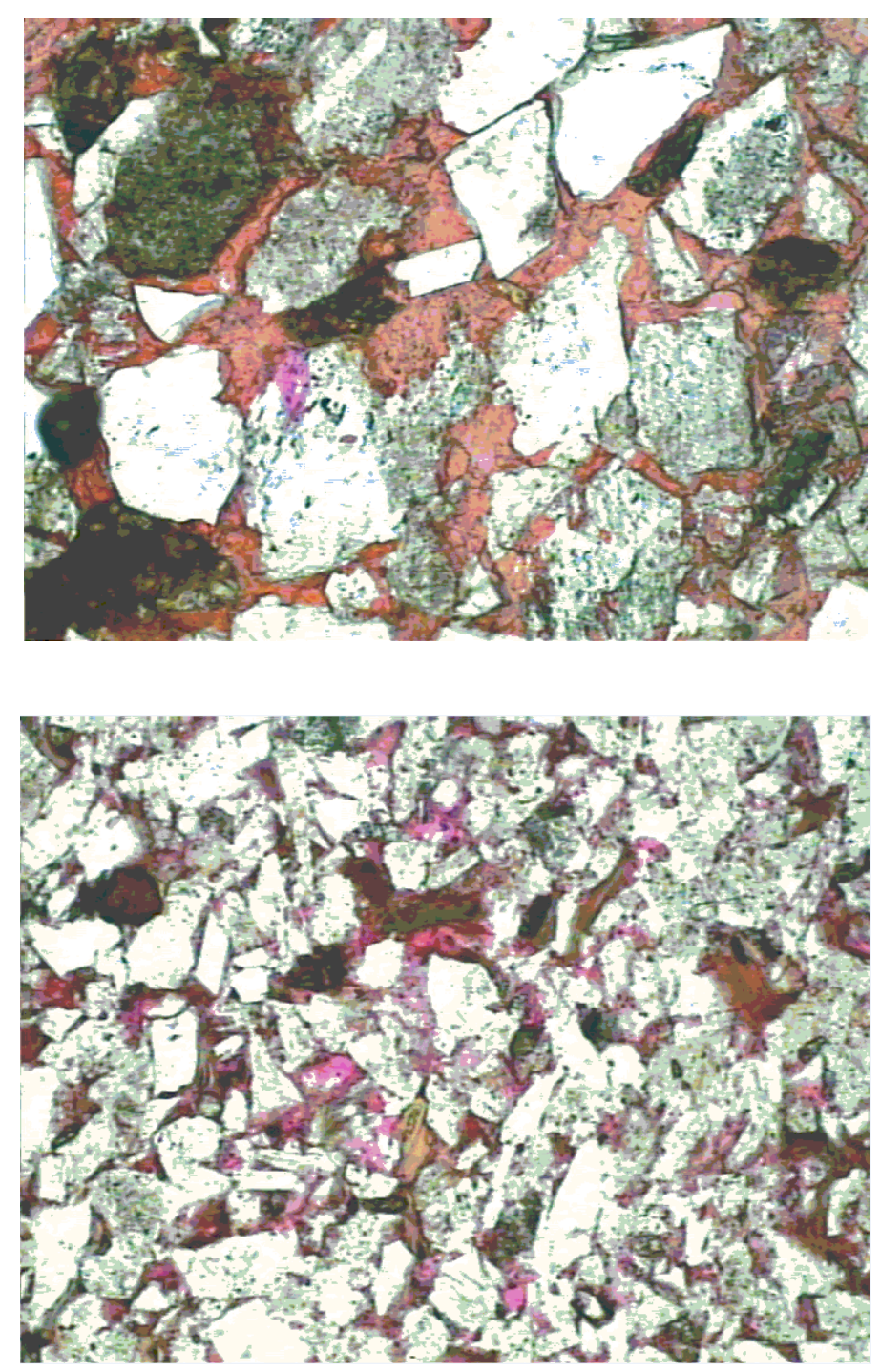

(a) Core image

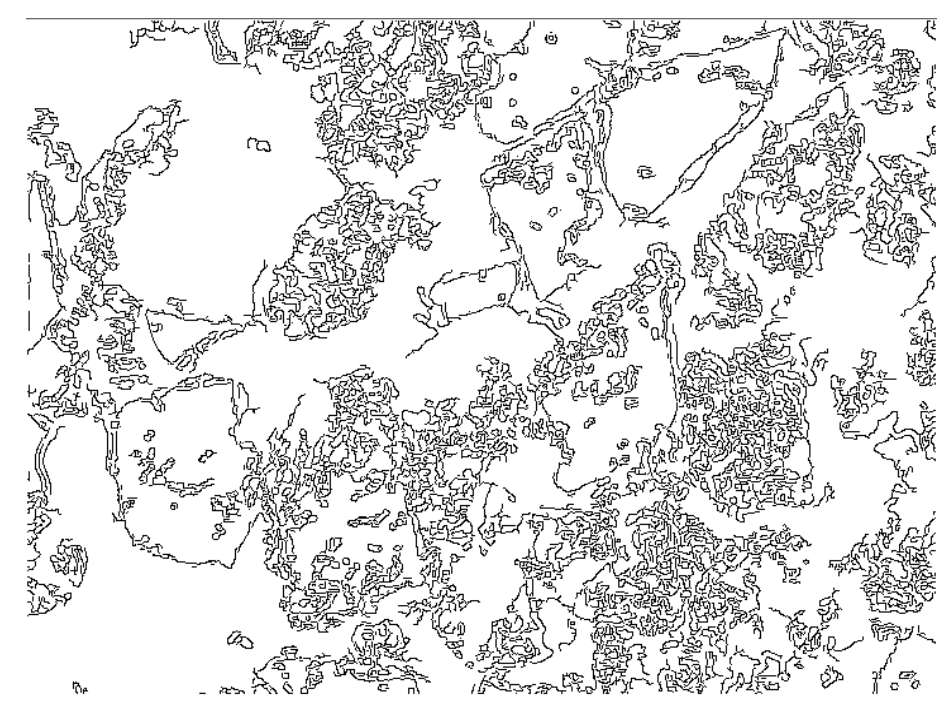

Fig. (2). Contd... 


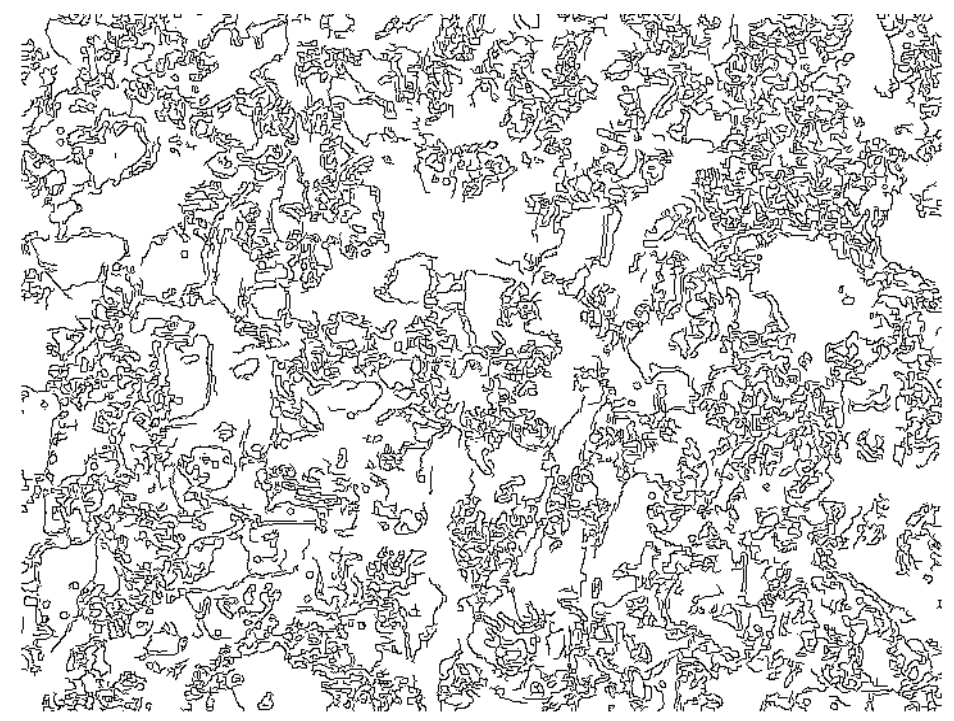

(b) Canny edge detection algorithm
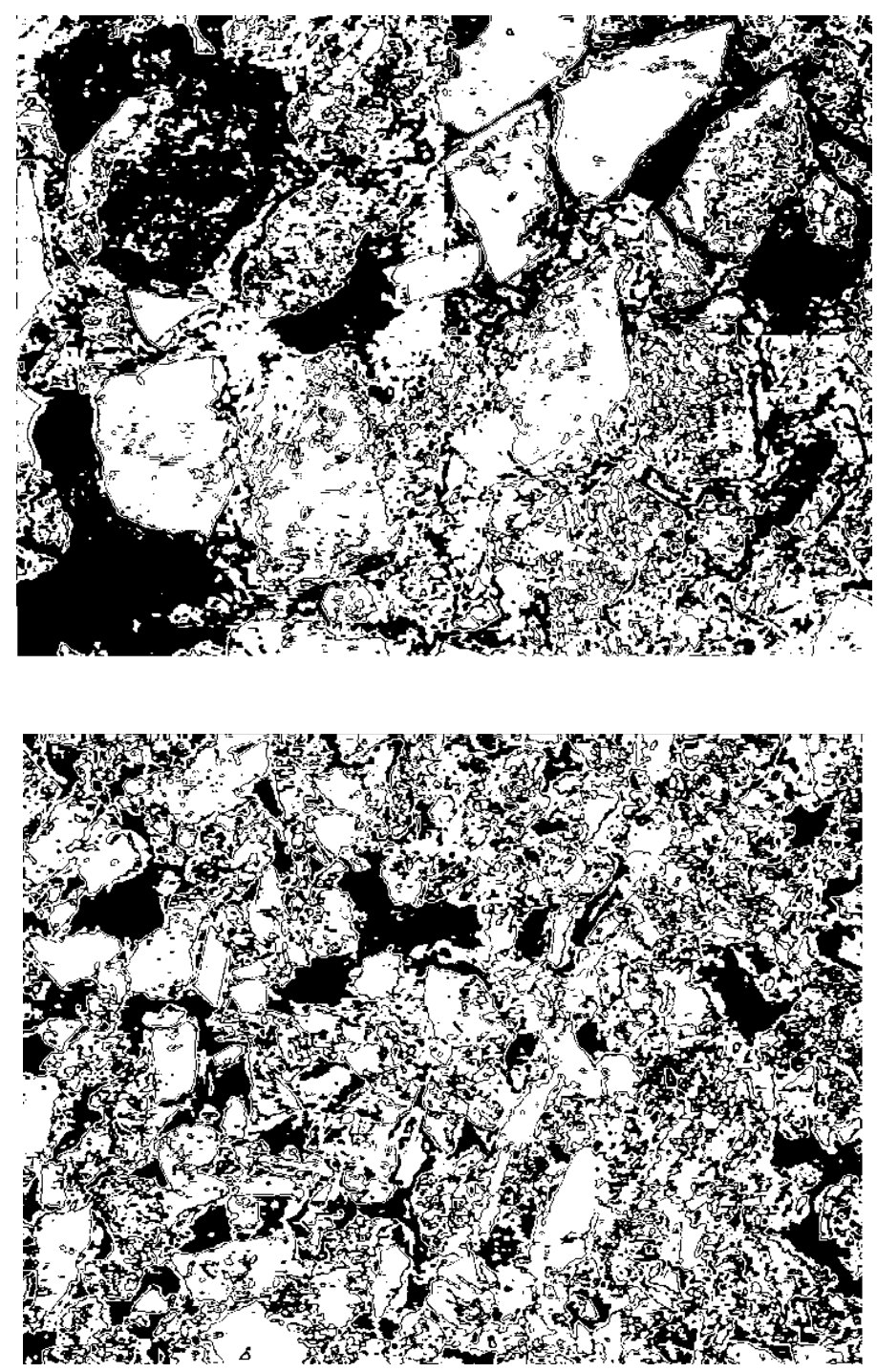

(c) Improved Canny edge detection algorithm

Fig. (2). Results comparison of two algorithms. 
represents the background region which is composed by the pixels whose gray level value range is between 0 and $t . B$ represents an object region which is composed by the pixel whose gray level value range is between $t$ and $K$.

\section{1) Establish the histogram of gray level}

Its gray-scale is $\mathrm{K}$. The occurrence probability $p_{i}$ of gray level value ' $i$ ' is defined by

$\mathrm{p}_{\mathrm{i}}=\frac{\mathrm{n}_{\mathrm{i}}}{\mathrm{M}}, \quad \mathrm{i}=0,1,2,3 \ldots, \mathrm{K}-1$

$\sum_{\mathrm{i}=0}^{\mathrm{K}-1} \mathrm{p}_{\mathrm{i}}=1, \sum_{\mathrm{i}=0}^{\mathrm{K}-1} \mathrm{n}_{\mathrm{i}}=\mathrm{M}$ ject

2) Calculate the probability of the background and the ob-

Where $\mathrm{p}_{\mathrm{A}}$ represents the occurrence probability of background and $\mathrm{p}_{\mathrm{B}}$ represents the occurrence probability of object. The corresponding formula is defined by

$\mathrm{P}_{\mathrm{A}}=\sum_{\mathrm{i}=0}^{\mathrm{t}} \mathrm{P}_{\mathrm{i}}, \mathrm{P}_{\mathrm{B}}=\sum_{\mathrm{i}=\mathrm{t}+1}^{\mathrm{K}} \mathrm{P}_{\mathrm{i}}$

3) Calculate the maximum between-cluster variance of $A$ region and $\mathrm{B}$ region

The average gray level of whole image is $\mathrm{u}_{\mathrm{T}} \cdot \mathrm{u}_{\mathrm{A}}$ and

$\mathrm{u}_{\mathrm{B}}$ are the average gray level value of the region $\mathrm{A}$ and region $B$ respectively. The between-cluster variance is $\sigma$. The corresponding formulas are defined by

$$
\begin{aligned}
& \mathrm{u}_{\mathrm{T}}=\sum_{\mathrm{i}=0}^{\mathrm{K}} \mathrm{ip}_{\mathrm{i}} \\
& \mathrm{u}_{\mathrm{A}}=\sum_{\mathrm{i}=0}^{\mathrm{t}} \frac{\mathrm{ip} \mathrm{P}_{\mathrm{i}}}{\mathrm{P}_{\mathrm{A}}}, \mathrm{u}_{\mathrm{B}}=\sum_{\mathrm{i}=\mathrm{t}+1}^{\mathrm{K}} \frac{\mathrm{ip} \mathrm{P}_{\mathrm{i}}}{\mathrm{P}_{\mathrm{T}}}=\mathrm{P}_{\mathrm{A}} \mathrm{u}_{\mathrm{A}}+\mathrm{P}_{\mathrm{B}} \mathrm{u}_{\mathrm{B}} \\
& \sigma^{2}=\mathrm{P}_{\mathrm{A}}\left(\mathrm{u}_{\mathrm{T}}-\mathrm{u}_{\mathrm{A}}\right)^{2}+\mathrm{P}_{\mathrm{B}}\left(\mathrm{u}_{\mathrm{T}}-\mathrm{u}_{\mathrm{B}}\right)^{2}
\end{aligned}
$$

4) Obtain the optimal thresholds $t_{1}$ and $t_{2}$.

The optimal thresholds are the gray level values which have the maximum between-cluster variance $\sigma$ of the background region $\mathrm{A}$ and the target region $\mathrm{B}$ in the image.

Step 6: Connect edge. The candidate edge points are selected and connected by recursive search.

\section{EXPERIMENTAL RESULTS AND ANALYSIS}

This paper improved the classical Canny algorithm by using the adaptive smooth filtering algorithm and adaptive threshold algorithm, and applied the improved Canny algorithm to the edge detection of the core image, as shown in Fig. (2). In Fig. (2), the part (a) is the two core images, in which the white and grey are rock and the red are rock pore for storing crude oil; the part (b) is the two extracted core edge images by the classical Canny algorithm; and the part (c) is the two extracted core edge images by the improved Canny algorithm of this paper. From the comparison between Fig. (2b) and Fig. (2c), it can be found that the adaptive Canny algorithm can improve the smoothness of image, the character of image edge, and the precision and accuracy of edge localization. In addition, the result processed by improved algorithm also can remove the image noise. The extracted edge image by the detection of improved algorithm contains richer details of edge and better contour information than the original image edge. The requirements of smooth noise and suppression the false edge are considered by the improved algorithm which can show the edge and pore structure of rock more clearly.

\section{CONCLUSION}

This paper proposed a core image edge detection algorithm based on improved Canny algorithm which combined the technique characteristics of improved adaptive smooth filtering algorithm with adaptive threshold algorithm. The final experiment results showed that the improved algorithm could retain weaker boundary and reflect the details of core crack, particles, and pore distribution more clearly. The detection results could provide more abundant information and were beneficial in explaining rock data compared with the traditional edge detection algorithm.

1) The paper mainly aimed at the process of smooth filtering and threshold selection to improve the traditional canny algorithm appropriately.

2) In this paper, the Gaussian filtering of the traditional Canny algorithm was replaced by the adaptive filtering. The best effect of iteratiion could be achieved by the optimal iteration algorithm which was used to select the iterations. In addition, the accurate edge information could be obtained by the algorithm which used adaptive method to automatically generate thresholds.

3) For different core image, the paper's algorithm needs to be researched and improved much further.

\section{CONFLICT OF INTEREST}

The author confirms that this article content has no conflict of interest.

\section{ACKNOWLEDGEMENTS}

This study was supported by Natural Science Foundation Project of Shaanxi Province of China No.2012JM8037.

\section{REFERENCES}

[1] H.D. Cheng, X.H. Jiang, Y. Sun, and J.L. Wang "Color image segmentation: advances and prospects". Pattern Recognition, vol. 34, pp. 2259-2281, 2001.

[2] E.J. Wharton, K. Panetta, S.S. Agaian. "Logarithmic edge detection with applications". Journal of Computers, vol. 3, pp. 3346-3351, 2008 . 
[3] Y. Zhang, and P.I. Rockett. "The Bayesian operating point of the Canny edge detector". IEEE Transactions on Image Processing, vol. 15, pp. 3409-3416, 2006.

[4] Y.G. Hong. "Human Action Identification Model Based on Improved Canny Operator and Neural Network", Computer Engineering and Application, vol. 49, pp. 157-158, 2013. (in Chinese)

[5] J. Zhao, W. Li, and Z.P. Hao. "Image edge detection based on improved canny operator and image morphology fusion". Microcomputer \& Its Applications, vol. 30, pp. 44-47, 2011. (in Chinese)

[6] Y. Furukawa, and J Ponce. "Carved visual hulls for image-based modeling". International Journal of Computer Vision, vol. 81, pp. 53-67, 2009.

[7] K.L. Hu, L.Y. Zhao, Z.H. Lin, and Y.G. Wang. "Improved method for extracting foreground based on self-adaptive threshold". Application Research of Computers, vol. 28, pp. 4727-4728, 2011. (in Chinese)

[8] J.M. Liang, and Z.X. Wei. "Research on improvement of the ostu algorithm". Jonrnal of Sichuan University of Science \& Engineering (Natural Science Edition), vol. 23, pp. 544-545, 2010. (in Chinese)

[9] Z.G. Qu, Y.H. Gao, P. Wang, P. Wang, X. Tanm and Z. Shen. "Contour detection improved by frequency domain filtering of gradient image". Science China (Information Sciences), vol. 57, pp. 13, 2014.

[10] A. Hornung, and L. Kobbelt. "Hierarchical Volumetric Multi-view Stereo Reconstruction of Manifold Surfaces Based on Dual Graph
Embedding", Proceedings of IEEE Conference on Computer Vision and Pattern Recognition. Washington D. C., USA: IEEE Computer Society, 2006 pp. 503-510.

[11] C.H. Esteban, and F. Schmitt. "Silhouette and stereo fusion for 3d object modeling". Computer Vision and Image Understanding, vol. 96, pp. 367-392, 2004.

[12] A. Zaharescu, E. Boyer, and R. Horaud. "Transformesh: a topology adaptive mesh-based approach to surface evolution", Proceedings of the $8^{\text {th }}$ Asia Conference on Computer Vision. Tokyo, Japan: Springer, 2007: 166-175.

[13] H. Wasaki, and H. Haneishi. "Gaussian-noise removal by an adaptive bilateral filter using perceptual noise sensitivity". Journal of The Society of Photographic Science and Technology of Japan, vol. 73, pp. 113-122, 2010.

[14] X.J. Jing, J.F. Li, and Y.Q. Xiong. "An adaptive smooth algorithm for still images". Journal of communication, vol. 23, pp. 9-10, 2002. (in Chinese)

[15] Y. He, Z Yuan, and F.Q. Cai. "Speckle suppression using anisotropic diffusion based on information-theoretic heterogeneity measurement". Acta Aeronautica et Astronautica Sinica, vol. 34, pp. 154-155, 2013. (in Chinese).

[16] Z.A.C. Barcelos, M. Boaventura, and E. Silva. "A well - balanced flow equation for noise removal and edge detection". IEEE Transactions on image processing, vol. 12, pp. 751-762, 2003.

Received: September 22, 2014

(C) Liu et al.; Licensee Bentham Open.

This is an open access article licensed under the terms of the Creative Commons Attribution Non-Commercial License (http://creativecommons.org/licenses/by-nc/3.0/) which permits unrestricted, non-commercial use, distribution and reproduction in any medium, provided the work is properly cited. 